scheme is advantageous for problems in which minimum mixing of the marks at each step is important.

5. Acknowledgements. This work was performed under the auspices of the U.S. Atomic Energy Commission.

The need for such a scheme was suggested by Professor D. H. Lehmer of the University of California at Berkeley.

University of California

Los Alamos Scientific Laboratory

Los Alamos, New Mexico

1. C. B. Tomprins, "Machine attacks on problems whose variables are permutations," Proceedings of Symposia in Applied Mathematics, v. VI, Numerical Analysis, McGraw-Hill, New York, 1956, pp. 195-211.

2. D. H. LeHMER "Teaching combinatorial tricks to a computer," Proceedings of Symposia in Applied Mathematics, v. X, Combinatorial Analysis, American Mathematical Society, Providence, R. I., 1960, p. 179-193.

\title{
Chebyshev Approximations to the Gamma Function
}

\section{By Helmut Werner and Robert Collinge}

In this note several Chebyshev approximations are given for the function $y=\Gamma(x+2)$ for $x$ in the $0 \leqq x \leqq 1.0$ range. The approximations were obtained from a table of $\Gamma(x+2)$, employing well-known methods as described in numerous papers; see for instance [1] and the literature quoted there. The table of $\Gamma(x+2)$ was calculated from the asymptotic expansion of $\log \Gamma(z)$ as given in [2] to provide data accurate to at least $10^{-21}$. Compare also [3].

The asymptotic expansion of $\ln \Gamma(z)$, is given by

$$
\ln \Gamma(z)=\left(z-\frac{1}{2}\right) \ln z-z+\ln \sqrt{2 \pi}+\Phi(z)
$$

where

$$
\Phi(z)=\sum_{r=1}^{n} \frac{(-1)^{r-1} B_{r}}{2 r(2 r-1)} \frac{1}{z^{2 r-1}}+R_{n}(z),
$$

and $B_{r}$ is the $r$ th Bernoulli number.

It can be shown [2] that for $z>0$ the value of $\Phi(z)$ always lies between the sum of $n$ terms and the sum of $(n+1)$ terms of the series, for all values of $n$. In terminating this series with the $n$th term the error $R_{n}(z)$ will be less than

$$
\frac{B_{n+1}}{2(n+1)(2 n+1)} \cdot \frac{1}{z^{2 n+1}} \text {. }
$$

By truncating $\Phi(z)$ at the 10th term it is easily shown that for values of $z \geqq 13$, the error in the expansion is less than $5.5 \times 10^{-22}$. We therefore replace $\Phi(z)$ by $\sum_{i=1}^{10} A_{i} / z^{2 i-1}$ and calculate $\ln \Gamma(z)$ for values of $z$ in the range $13 \leqq z \leqq 14$.

Received July 25, 1960. 
T'ABLe 1

Table of Coefficients

\begin{tabular}{|c|c|c|c|c|}
\hline * & 7 & \multicolumn{2}{|l|}{8} & 10 \\
\hline$\overbrace{\max }^{(n)}$ & $0.23 \times 10^{-i}$ & \multicolumn{2}{|l|}{$0.16 \times 10^{-7}$} & $0.74 \times 10^{-11}$ \\
\hline $\begin{array}{r}\nu=0 \\
1 \\
2 \\
3 \\
4 \\
5 \\
6 \\
7 \\
8 \\
9 \\
10\end{array}$ & $\begin{array}{ll}0.99999 & 99758 \\
0.42278 & 74605 \\
0.41177 & 41955 \\
0.08211 & 17404 \\
0.07211 & 01567 \\
0.00445 & 11400 \\
0.00515 & 89951 \\
0.00160 & 63118\end{array}$ & $\begin{array}{rr}0.99999 & 999 \\
0.42278 & 436 \\
0.41183 & 929 \\
0.08159 & 034 \\
0.07416 & 009 \\
0.00007 & 559 \\
0.01033 & 206 \\
-0.00157 & 800 \\
0.00079 & 624\end{array}$ & $\begin{array}{ll}98 & 452 \\
02 & 730 \\
35 & 920 \\
49 & 474 \\
15 & 535 \\
34 & 181 \\
35 & 065 \\
74 & 635 \\
04 & 760\end{array}$ & $\begin{array}{rrr}0.99999 & 99999 & 9269 \\
0.42278 & 43369 & 6202 \\
0.41184 & 02517 & 9616 \\
0.08157 & 82187 & 8492 \\
0.07423 & 79076 & 0629 \\
-0.00021 & 09074 & 6731 \\
0.01097 & 36958 & 4174 \\
-0.00246 & 67479 & 8054 \\
0.00153 & 97681 & 0472 \\
-0.00034 & 42342 & 0456 \\
0.00006 & 77105 & 7117\end{array}$ \\
\hline$n$ & \multicolumn{2}{|c|}{13} & \multicolumn{2}{|r|}{15} \\
\hline$e_{\max }^{(n)}$ & \multicolumn{2}{|c|}{$0.96 \times 10^{-16}$} & \multicolumn{2}{|r|}{$0.97 \times 10^{-16}$} \\
\hline $\begin{array}{r}\nu=0 \\
1 \\
2 \\
3 \\
4 \\
5 \\
6 \\
7 \\
8 \\
9 \\
10 \\
11 \\
12 \\
13 \\
14 \\
15\end{array}$ & \multicolumn{2}{|c|}{$\begin{array}{rrrrr}0.99999 & 99999 & 99990 & 44 \\
0.42278 & 43351 & 02334 & 79 \\
0.41184 & 03301 & 66781 & 29 \\
0.08157 & 69261 & 24155 & 46 \\
0.07424 & 89154 & 19444 & 74 \\
-0.00026 & 61865 & 94953 & 06 \\
0.01114 & 97143 & 35778 & 93 \\
-0.00283 & 64625 & 20372 & 82 \\
0.00206 & 10918 & 50225 & 54 \\
-0.00083 & 75646 & 85135 & 17 \\
0.00037 & 53650 & 52263 & 07 \\
-0.00012 & 14173 & 48706 & 32 \\
0.00002 & 79832 & 88993 & 83 \\
-0.00000 & 30301 & 90810 & 28\end{array}$} & \multicolumn{2}{|c|}{$\begin{array}{rrrr}0.99999 & 99999 & 99999 & 9032 \\
0.42278 & 43350 & 98518 & 1178 \\
0.41184 & 03304 & 21981 & 4831 \\
0.08157 & 69194 & 01388 & 6786 \\
0.07424 & 90079 & 43401 & 2692 \\
-0.00026 & 69510 & 28755 & 5266 \\
0.01115 & 38196 & 71906 & 6992 \\
-0.00285 & 15012 & 43034 & 6494 \\
0.00209 & 97590 & 35077 & 0629 \\
-0.00090 & 83465 & 57420 & 0521 \\
0.00046 & 77678 & 11496 & 4956 \\
-0.00020 & 64476 & 31915 & 9326 \\
0.00008 & 15530 & 49806 & 6373 \\
-0.00002 & 48410 & 05384 & 8712 \\
0.00000 & 51063 & 59207 & 2582 \\
-0.00000 & 05113 & 26272 & 6698\end{array}$} \\
\hline$n$ & \multicolumn{2}{|c|}{17} & \multicolumn{2}{|r|}{18} \\
\hline$e_{\max }^{(n)}$ & \multicolumn{2}{|c|}{$0.10 \times 10^{-17}$} & \multicolumn{2}{|r|}{$0.10 \times 10^{-18}$} \\
\hline $\begin{array}{r}\nu= \\
1 \\
2 \\
3 \\
4 \\
5 \\
6 \\
7 \\
8 \\
9 \\
10 \\
11 \\
12 \\
13 \\
14 \\
15 \\
16 \\
17 \\
18\end{array}$ & 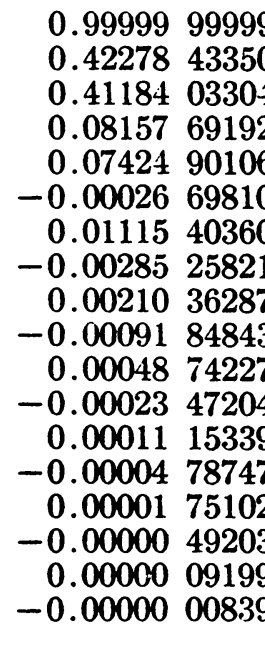 & $\begin{array}{lll}99999 & 99901 & 2 \\
98467 & 79580 & 6 \\
26367 & 20638 & 1 \\
50260 & 90508 & 9 \\
80090 & 41696 & 9 \\
33348 & 38176 & 8 \\
24034 & 39169 & 2 \\
44619 & 65607 & 6 \\
02459 & 83329 & 2 \\
69099 & 08014 & 2 \\
94476 & 75810 & 4 \\
01891 & 94985 & 9 \\
51966 & 59947 & 0 \\
98383 & 44672 & 4 \\
72717 & 90508 & 0 \\
75090 & 42313 & 2 \\
15640 & 71621 & 4 \\
94049 & 59039 & 7\end{array}$ & $\begin{array}{r}0.99 \\
0.42 \\
0.41 \\
0.08 \\
0.07 \\
-0.00 \\
0.01 \\
-0.00 \\
0.00 \\
-0.00 \\
0.00 \\
-0.00 \\
0.00 \\
-0.00 \\
0.00 \\
-0.00 \\
0.00 \\
-0.00 \\
0.00\end{array}$ & $\begin{array}{lllll}99999 & 99999 & 99990 & 02 \\
43350 & 98467 & 21319 & 64 \\
4 & 03304 & 26430 & 62304 & 23 \\
69192 & 47528 & 84581 & 87 \\
4 & 90107 & 42094 & 91715 & 38 \\
69818 & 88740 & 38315 & 07 \\
40438 & 29069 & 91793 & 28 \\
4 & 26318 & 64702 & 11862 & 89 \\
38579 & 20672 & 20524 & 09 \\
92675 & 95039 & 95026 & 11 \\
9 & 94361 & 06998 & 14458 & 34 \\
36428 & 33752 & 63647 & 10 \\
73283 & 10224 & 09396 & 51 \\
43183 & 86280 & 13508 & 99 \\
28140 & 41153 & 66022 & 75 \\
80523 & 43363 & 48309 & 46 \\
21741 & 77495 & 45532 & 64 \\
03889 & 70057 & 38769 & 55 \\
00339 & 81801 & 01810 & 43\end{array}$ \\
\hline
\end{tabular}


For the convenience of the reader the $A_{i}$ coefficients are quoted below, to 25 significant figures.

$$
\begin{aligned}
& A_{1}=0.08333333333333333333333333 \\
& A_{2}=-0.002777777777777 \quad 777777777778 \\
& A_{\mathbf{z}}=\quad 0.0007936507936507936507936508 \\
& A_{4}=-0.0005952380952380952380952381 \\
& A_{6}=\quad \begin{array}{lllll}
0.00084 & 17508 & 41750 & 8417508417 & 508
\end{array} \\
& A_{6}=-0.001917526917526917526917527 \\
& A_{7}=0.006410256410256410256410256 \\
& A_{3}=-0.02955065359477124183006536 \\
& A_{9}=0.1796443723688305731649385
\end{aligned}
$$

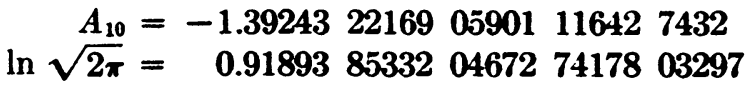

A triple precision logarithm routine was used to evaluate $\ln z$, and then an exponential routine to calculate $\Gamma(z)=e^{\ln \Gamma(z)}$. Each of these routines produces results accurate to at least 24 significant digits.

After obtaining a table of $\Gamma(z)$ for $z$ in the range $13 \leqq z \leqq 14$, we made use of the recursion formula $\Gamma(z+1)=z \Gamma(z)$ in order to obtain a table of $\Gamma(x+2)$ for $x$ in the range $0 \leqq x \leqq 1.0$.

From the tests made on the results obtained, the values of $\Gamma(x+2)$ were shown to be accurate to at least 21 significant figures.

Several Chebyshev approximations have been calculated to provide varying degrees of accuracy. Let

$$
\Gamma(2+x)=\sum_{\nu=0}^{n} a_{\nu}^{(n)} x^{p}+\epsilon_{n}(x)
$$

and

$$
\epsilon_{\max }^{(x)}=\max _{0 \leq x \leq 1}\left|\epsilon_{n}(x)\right| \text {. }
$$

Table 1 gives the coefficients $a_{v}^{(n)}$ for $n=7,8,10,13,15,17,18$ together with the corresponding $\dot{\epsilon}_{\max }^{(n)}$.

Professional Services Department

Burroughs Corporation

460 Sierra Madre Villa

Pasadena, California

1. E. L. STIEFel, "Numerical methods of Tchebycheff approximation," On Numerical Approximation by R. E. Langer, The University of Wisconsin Press, Madison, 1959, p. 217-232. 251-253.

3. M. E. ShERRY \& S. FULDA, "Calculation of gamma functions to high accuracy," $M T A C$, v. 13, 1959, p. 314-315.

4. H. S. Uther, "Log $\pi$ and other basic constants," Proc. Nat. Acad. Sci. U. S. A., v. 24, 1938, p. 23-30.

5. H. S. UHLER, "The coefficients of Stirling's series for $\log \Gamma(x)$," Proc. Nat. Acad. Sci. U. S. A., v. 28, 1942, F. 59-62. 